\title{
The Research of Dynamical Model of Corn Cob
}

\author{
Linglin Zhang, Lihua Zhang ${ }^{\mathrm{C}}$, Jiamei He, Bo Wang \\ College of mechanical and electrical engineering \\ Sichuan Agricultural University \\ Ya'an, China
}

\begin{abstract}
Corn cob dry is the key link of making corn cob powder. In order to ensure the quality of crushed corn cob, the research analyzes the features of hot-air dry of corn cob according to the experiment of corn cob hot-air drying. And the passage inquiries into the influence of different temperatures, wind speeds and the weight of load to the procedure of hot-air dry, in order to obtain the regular pattern of dehydration of hot air dry and to establish and verify dynamical model of corn cob hot air dry according to the experiment results. Results have showed that the best model of corn cob hot-air dry is model Page by fitting and analyzing. Fitting equation $\ln (-\ln M R)=-0.0020-0.0795 \mathrm{~A}-$ $0.0023 B-0.0993 E+(0.0004+0.0146 A+0.0147 A-$ $0.0004 \mathrm{~B}+0.0184 \mathrm{E}$ ) Int can better describe the procedure of corn cob hot air dry and precisely forecast the water content of and dehydrating rate of corn cob at each stage.
\end{abstract}

Keywords-Corn cob; Hot air dry; Dynamical model; Parameter

\section{INTRODUCTION}

Corn cob is the cob core of threshed maize. At present, China's annual maize production is about 1.2 tones. Corn cob accounts for corn weight about $20 \% \sim 30 \%{ }^{[1]}$. Corn cob is enriched with nutrients and the main components include $54.5 \%$ sugar, $2.2 \%$ crude protein, $0.4 \%$ crude fat, $29.7 \%$ crude fiber and $1.2 \%$ mineral, etc. ${ }^{[2,3]}$ Corn cob hotair dry is the crucial technology of deep processing. Therefore, deep research on corn cob drying technology has certain practical significance.

Currently, drying technology includes: Hot-air dry, Microwave dry, Freeze dry and Microwave vacuum dry, etc. Hot-air dry strongly adaptability to various materials, and equipment and processing costs are relatively low. In recent years, study on hot-air drying dynamics model of many agricultural products have been reported in the literature ${ }^{[4-10]}$. However, there are no reports on the study of corn cob hot-air drying dynamics model. The research uses hot-air to sheet of thin layer drying of corn cob and discusses effects of different thickness, air temperatures and wind speeds on the drying. Then use regression fitting methods to establish the drying dynamics model.

\section{MATERIALS AND METHODS}

\section{A. Materials}

Fresh corn cob, whose initial water content reaches $70 \% \sim 75 \%$. Retain moisture in the fresh keeping bag and keep it in cold storage in the refrigerator. Pretreatment on the test materials: corn cob $\rightarrow$ select $\rightarrow$ remove both ends $\rightarrow$ slice up $\rightarrow$ tray

\section{B. The main equipment}

Device and equipment: DG100D digital tunnel drying device (Zhejiang SUPCON Scientific Instrument Co., Ltd.); $200 \times 0.01 \mathrm{~mm}$ digital Vernier caliper (USA bunker, the precision is $0.01 \mathrm{~mm}$ ); GHG-9101-3SA type electric thermostatic drying oven (Shanghai Sanfa Scientific Instrument Co., Ltd); OHAUS-AR522CN electronic precision balance (Ohaus instruments (Shanghai) Co., Ltd. manufacturing index value of $0.01 \mathrm{~g}$ ).

\section{Drying test}

Set drying device according to the test conditions ,after the stability of temperature and wind velocity, weigh the corn chips, lay the chips on the drying plate, cover the whole drying area, then put it into drying device, record the time and mass. The mass becomes unchanged, stop test. Finally, the material at $150^{\circ} \mathrm{C}$ has constant mass, use the corn cob dry mass to data processing.

\section{Test Index}

(1)Moisture content (d.b.) $\mathrm{W}_{\mathrm{t}}$ : according to(1).

$W_{t}=\frac{M_{\mathrm{t}}-M_{g}}{M_{\mathrm{g}}} \times 100 \%$

$\mathrm{M}_{\mathrm{t}}$ - - The mass of corn cob at $t$ moment, g;

$\mathrm{M}_{\mathrm{g}}$ - Dry weight of corn cob, $\mathrm{g}$;

(2)The drying velocity $\mathrm{v}\left[\mathrm{g} /\left(\mathrm{m}^{2} . \mathrm{s}\right)\right]$ : Removed water mass in the unit area in the unit of time ${ }^{[11]}$, according to (2).

$v=\frac{\Delta m}{\Delta t}$

$\Delta m$ - Changes in mass between two adjacent measurements; $\Delta t$-Time interval of two adjacent measurements, $\mathrm{s}$; $\mathrm{A}$-Drying contact area, $\mathrm{m}^{2}$. 


\section{CONCLUSION AND ANALYSIS}

\section{A. Effects of drying conditions on the drying process}

1) Effects of different wind temperatures on the drying process

To change the default, adjust the template as follows. Setting the mass of $70 \mathrm{~g}$, the wind speed of $1.5 \mathrm{rn} / \mathrm{S}$, and the temperatures respectively are $60^{\circ} \mathrm{C}, 70^{\circ} \mathrm{C}, 80^{\circ} \mathrm{C}$.In the case of unchanged wind speed, the drying curves and drying rate curves of flake corn cob are shown in Fig .1 and Fig .2.

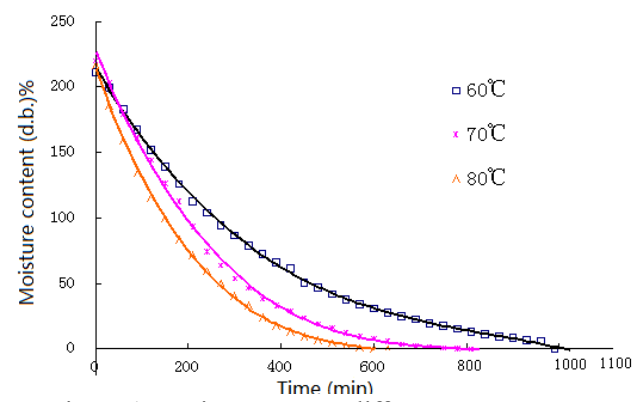

Figure 1. Drying curves at different temperatures

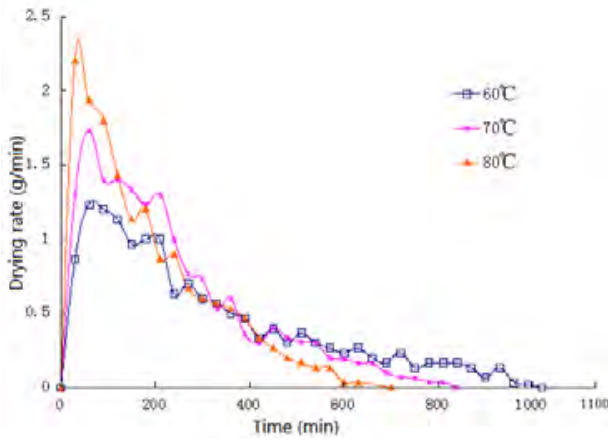

Figure 2. Drying rate curves at different temperatures

From the curves in Fig .1, along with the increase of temperature, the drying curve become steeper, after the same drying time its moisture content is lower, so it takes less time to reach safe moisture content. In the end stage of drying, drying time is longer, the difficulty of dehydration is greater, and drying becomes more difficult. Therefore improving the drying air temperature benefits the process. As shown in Fig .2, corn cob hot-air drying process can be divided into the acceleration stage and deceleration stage, and the constant speed stage is not obvious, the drying process is mostly in the deceleration rate period, this is because the heat required for the evaporation of water must be gradually transferred from the materials' surface to the inside, in the process of drying, moisture in the outer layer of corn core first evaporates, outward migration rate of the inner water is less than the water loss rate of materials' surface, the outer layer of corn cob soon becomes dry, the resistance increases when inner water transfer outwards, leading to significant reduction of the water evaporation. Therefore, most of the corn cob drying process is in the deceleration rate period.
2) Effects of different wind speeds on the drying process

At $40{ }^{\circ} \mathrm{C}$, the mass of $50 \mathrm{~g}$, the wind speeds are respectively $0.56 \mathrm{~m} / \mathrm{s}, 1.15 \mathrm{~m} / \mathrm{s}, 1.74 \mathrm{~m} / \mathrm{S}$. The drying curves and drying rate curves of flake corn cob are shown in Fig 3 and Fig .4.

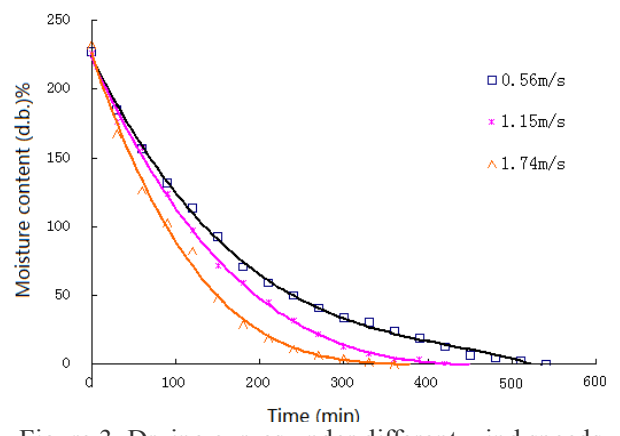

Figure 3. Drying curves under different wind speeds

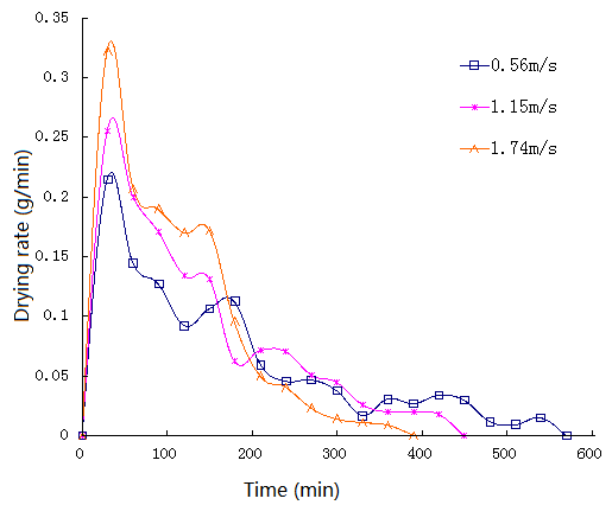

Figure 4. Drying rate curves under different wind speeds

As shown in Fig .3, the greater the wind speed, the lower the water content rate of material during the same time, the reason is that the greater the wind speed, humidity difference between air and material is bigger, at the same time, heat and mass transferring boundary layer got thinner, heat transfer coefficient and mass transfer coefficient increase, the drying rate is greater. Therefore, improving the wind speed is favorable to the drying process, but the greater the wind speed, the greater the energy loss. As shown in Fig .4, the greater the wind speed, the shorter the acceleration stage, constant speed stage is shorter, the critical moisture content is higher, the lower the equilibrium moisture content. The drying rate is affected by wind speed greatly in constant speed stage and increases when the wind speed increases, but its growth rate decreases gradually. Higher wind speed, higher air volume, transport and heating required more power, exhaust takes away more heat, higher energy consumption, lower thermal efficiency; When wind speed is $1.15 \mathrm{~m} / \mathrm{s}$ or $1.74 \mathrm{~m} / \mathrm{s}$, corn cob drying growth rate has been significantly reduced, wind speed should be $1.15 \mathrm{~m} / \mathrm{s}$.

3) Effects of different mass on drying process At $40^{\circ} \mathrm{C}$, the wind speed is $1.5 \mathrm{~m} / \mathrm{s}$, the mass of meterial is respectively $30 \mathrm{~g}, 90 \mathrm{~g}$ and $150 \mathrm{~g}$. The drying curves and drying rate curves are shown in Fig .5 and Fig .6. 


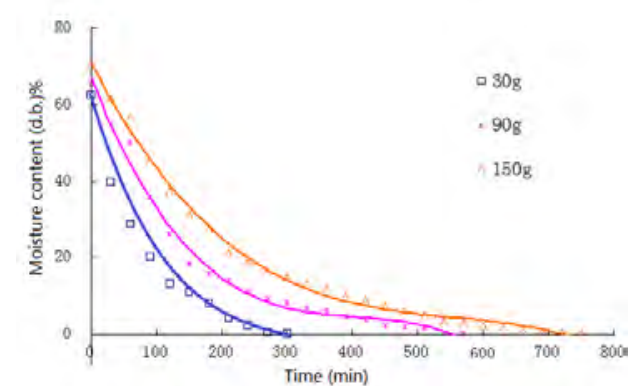

Figure 5. Drying curves of different mass

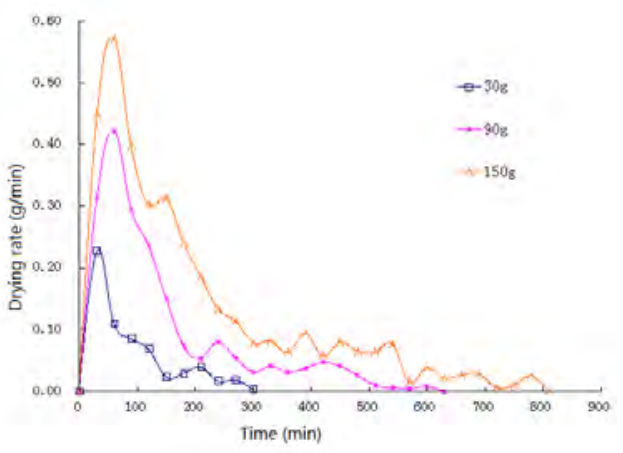

Figure 6. Drying rate curves of different mass

From Fig .5 and Fig .6, material piles thicker (loading is larger), the drying rate is slower. Thick material will extend drying time, this is because as the loading increases, more moisture needs to be removed in the drying process, when the air temperature and wind speed are under the same condition, the ability to put out the water of evaporation is certain, which leads to the drying time expanding; Multiple layers can reduce the contact area with the drying media and is not conductive to the evaporation of water in the center of material, resulting in dry rate decreasing. Material's quantity is too small, and it will increase the energy consumption. Therefore, material loading should be about $90 \mathrm{~g}$.

\section{B. Corn cob hot-air drying test model}

1) Select and confirm of the corn cob mathematical drying test model

The drying process of material is a very complex and relatively unstable heat and mass transfer process. The establishment of model plays an important role in the study of drying characteristics and prediction of drying parameters ${ }^{[12]}$. Nowadays, the research has summed up commonly used mathematical model to describe the material's drying process.

\section{TABLE II DRYING MATHEMATICAL MODEL}

\begin{tabular}{|c|c|c|}
\hline $\begin{array}{l}\text { Single diffusion } \\
\text { model }\end{array}$ & $\mathrm{MR}=\operatorname{Aexp}(-\mathrm{Kt})$ & $\ln (\mathrm{MR})=\ln \mathrm{A}-\mathrm{kt}$ \\
\hline Page equation & $M R=\exp \left(-K t^{n}\right)$ & $\ln (-\ln (\mathrm{MR}))=\ln \mathrm{k}+\mathrm{N} \ln t$ \\
\hline $\begin{array}{l}\text { Exponential } \\
\text { model }\end{array}$ & $M R=\exp (-K t)$ & $\ln (\mathrm{MR})=-\mathrm{kt}$ \\
\hline
\end{tabular}

t: Drying time, min; K: Drying rate constant; A: Pending rate coefficient; n: Ming index; MR: Moisture ratio.

$$
M R=\frac{M_{\mathrm{t}}-M_{e}}{M_{0}-M_{e}}
$$

Because of dry material equilibrium moisture content is too small to obtain from the test, so MR is simplified to

$$
M R=\frac{M_{\mathrm{t}}}{M_{0}}
$$

According to (5), calculate MR of corn cob in the drying process in this experiment.

The curves $-\ln M R$ - $t$ and $\ln (-\ln M R)-\ln t$ in different temperatures and wind speeds are drawn according to experimental data, as shown in Fig .7 and Fig .8.

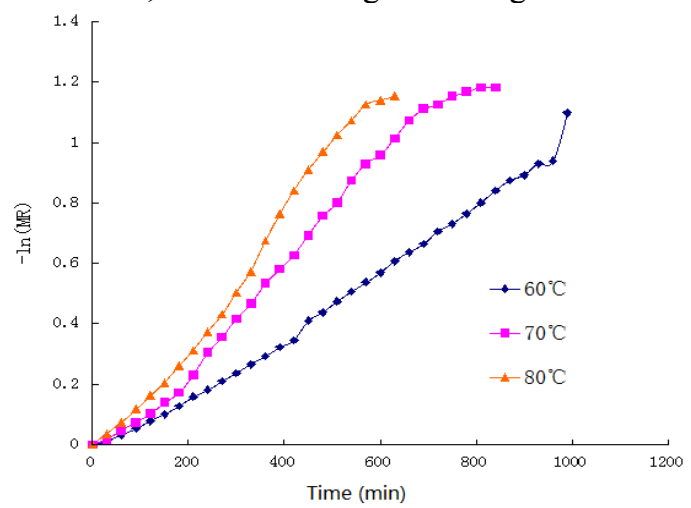

a. Different temperatures

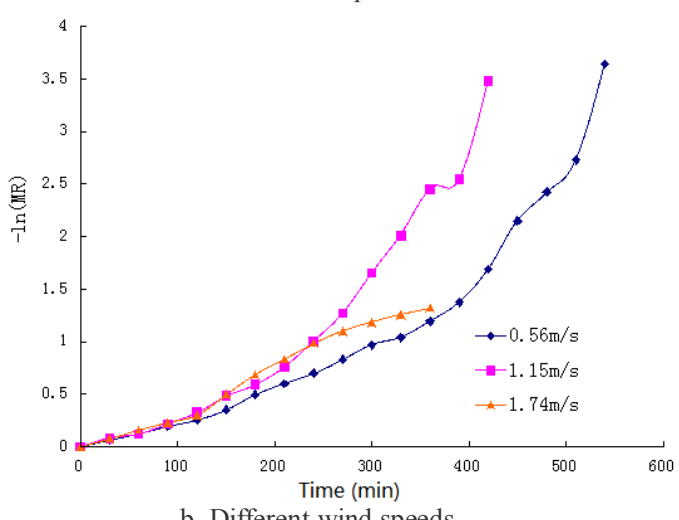

b. Different wind speeds

Figure 7. -lnMR--t curves under different temperatures and wind speeds

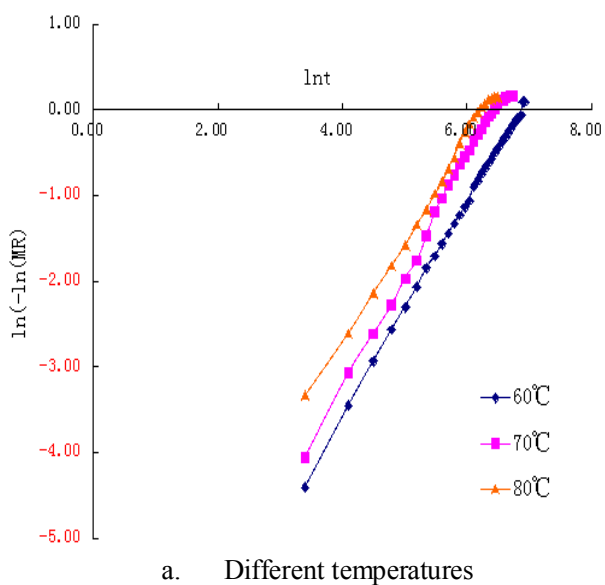




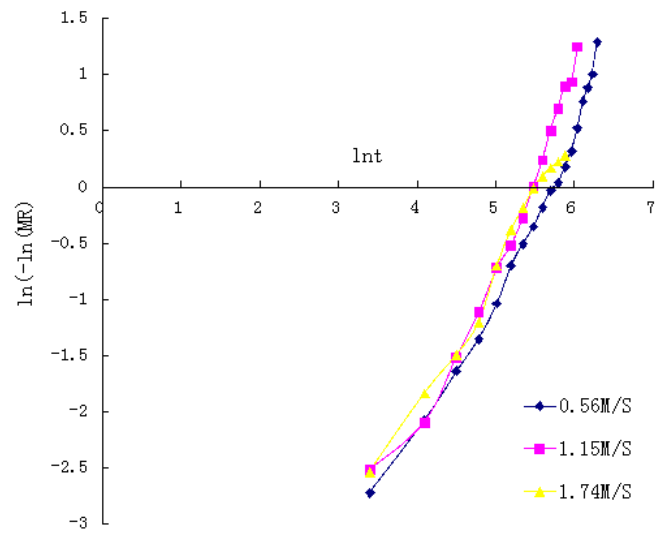

b. Different wind speeds

Figure8: $\ln (-\ln M R)$ - $\ln$ c curves under different temperatures and wind speeds

Seen from Figure 7, the slope at each point of the curve is different, it means that the $\ln (\mathrm{MR})$ has a nonlinear relationship with $t$, single diffusion model and exponential model are not suitable for hot-air drying corn cob model. Seen from figure 8 , the $\ln (-\ln M R)$ has a linear relationship with $\ln t$, which indicates that corn cob drying process can be described by Page equation. At different temperatures and under different wind speeds, curves of relationship of $\ln (-\ln M R)$ and $\ln t$ parallel, indicating that different temperatures and wind speeds have significant effects on the drying model. Therefore, choose the Page model as a dynamic model of corn cob dry.

$$
\begin{aligned}
& \ln r=a+b A+c B+d E \\
& N=e+f A+g B+\mathrm{h} E
\end{aligned}
$$

There is

$$
\ln (-\ln M R)=a+b A+c B+d E+(e+f A+g B+h E) \ln t
$$

A-temperature, ${ }^{\circ} \mathrm{C}$; B-wind speed, $\mathrm{m} / \mathrm{s}$; E-mass, g; a, b, c, d, e, f, g, h-undetermined coefficients.

The data is processed with MATLAB, the undetermined coefficients can be obtained during corn cob hot-air drying, and then the dynamic model is:

$$
\begin{aligned}
& \ln (-\ln M R)=-0.0020-0.0795 A-0.0023 B-0.0993 E+ \\
& (0.0004+0.0147 A+0.0004 B+0.0184 E) \ln t
\end{aligned}
$$

\section{2) Statistical test of model}

The coefficient of determination R2 $=0.9979$, the fitting effect is good. Therefore, this model can be used as a mathematical model of hot-air drying of corn cob. The model can be used to accurately predict the changes of moisture content and drying rate of corn cob in hot-air dry in different drying conditions.

\section{Verification of dynamical model}

Select a set of experiments to further validate the accuracy of the model. Test conditions: At $400{ }^{\circ} \mathrm{C}$, wind speed $0.56 \mathrm{~m} / \mathrm{s}$, drying mass $50 \mathrm{~g}$. Compare the actual value and the prediction value, as shown in Fig .9. The two curves are identical, it indicates that the Page equation can accurately response regularity of change of water and can be used to describe the process of hot-air dry of corn cob.

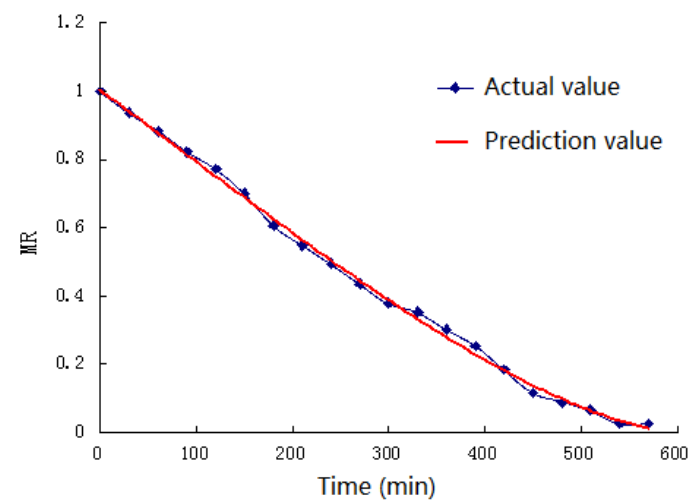

Figure 9: MR actual values and predicted values of Page model

\section{CONCLUSIONS}

(1) The corn cob drying is the same as traditional drying, divided into acceleration, constant rate and deceleration rate 3 stages.

(2) The influence of drying temperature and wind velocity on the drying process is significant. The higher the temperature, the greater the wind speed, the faster the average drying speed, but the quality is worse, corn cob may be coked by wind; Conversely, lower temperature and slower wind speed make the drying time longer and the drying quality better, but the cost is high. Thus it is necessary to control the drying temperature and choose appropriate drying speed.

(3) Comparing 3 common drying models, the best model of corn cob drying is Page model fitting equation:

$$
\begin{aligned}
& \ln (-\ln M R)=-0.0020-0.0795 A-0.0023 B-0.0993 E+ \\
& (0.0004+0.0147 A+0.0004 B+0.0184 E) \ln t
\end{aligned}
$$

From the experimental results, we can better predict the change of corn cob hot-air drying rate and moisture content at each stage, and provide a theoretical basis for the drying process of hot-air dry of corn cob.

\section{ACKNOWLEDGEMENTS}

Corresponding Author: Lihua Zhang, Professor, Master Instructor. Research Direction: Machine and equipment for agriculture products processing. Email: zhanglihua69@126.com.

\section{REFERENCES}

[1] Jingtao Yang, Jingquan Yu. The processing and application of corn cob [J]. New rural technology.2010,8:26-27.

[2] Yan Liu. Basic study on the comprehensive utilization of corn cob [D].JLU.2009:16-18. 
[3] Wenhua Wang, Yongmei Feng, Yan Sun, et al. Study on the adsorption of lead in iste water from corn cob[J]. JLU. Water treatment technology.2004, 30(2):95-98.

[4] Hongyi Chen, Manquan Zhao, Ping Li. Progressing research of drying technology of agricultural products[C]. JLU[C].CSAE. 2011 Symposium

[5] Jianjun Zhang, Haixia Wang, Yongchang Ma. Study on hot-air drying properties of Capsicum.Chinese society of Agricultural Engineering[J],2008,24(3):298-301.

[6] Guilan Peng, Wenfu Wu, Xiaoguang Chen, et al. The establishment of radish thin-layer drying experiment and mathematical model[J]. Journal of agricultural machinery,2005,36(3):79-81,94.

[7] Yan Huang, Jianli Huang, Baodong Zheng. The drying characteristics and kinetics model of microwave vacuum Tremella [J]. Chinese society of Agricultural Engineering, 2010,26(4):362367.
[8] Aishi Zhu, Kai Xia. Study on drying kinetics of thin layer of hotair bottle gourd[J].Chinese society of Agricultural Engineering, 2011, 27(01): 365-369.

[9] Xina Lin, Xiangyou Wang. Establishment and evaluation of apple slices of infrared radiation drying model[J]. Journal of agricultural machinery,2010,41(6):128-132.

[10] Lihua Zhang, Bo Liu, Taotao Liu, Guanghui Li. Study on the microwave drying characteristics and kinetics of ginkgo fruit intermittent model[J]. Science and technology of food industry,2013,34(18):127-131.

[11] Xiaoping Li, Mengmeng Wang, Xiaoli Lu. Study on hot-air drying characteristics and mathematical model of aging sweet potato[J]. Food and machinery,2007,23(05):119-121,142.

[12] Lihua Zhang, Wen Zhang, Zhenzhen Lu, Zhongming Xu. Distiller's grain microwave drying characteristics and dynamical model of intermittent[J].Food Science,2010,33(01):87-92. 